\title{
1 Intergenerational epigenetic inheritance in reef-building corals
}

2 Yi Jin Liew ${ }^{1, \dagger}$, Emily J. Howells ${ }^{2, \dagger}$, Xin Wang ${ }^{1}$, Craig T. Michell ${ }^{1}$, John A. Burt ${ }^{2}$, Youssef

3 Idaghdour ${ }^{2}$, Manuel Aranda ${ }^{1, *}$

\section{Affiliations}

$5 \quad{ }^{1}$ King Abdullah University of Science and Technology (KAUST), Red Sea Research Center

6 (RSRC), Biological and Environmental Sciences \& Engineering Division (BESE), Thuwal,

7 Saudi Arabia.

$8{ }^{2}$ Center for Genomics and Systems Biology, New York University Abu Dhabi, Abu Dhabi,

9 United Arab Emirates.

$10 *$ Correspondence to: manuel.aranda@kaust.edu.sa (M.A.)

11 Contributed equally

12 Keywords: Epigenetics, DNA methylation, coral, cnidarian, intergenerational inheritance. 
Main

The notion that intergenerational or transgenerational inheritance operates solely through genetic means is slowly being eroded: epigenetic mechanisms have been shown to induce heritable changes in gene activity in plants ${ }^{1,2}$ and metazoans ${ }^{1,3}$. Inheritance of DNA methylation provides a potential pathway for environmentally induced phenotypes to contribute to evolution of species and populations ${ }^{1-4}$. However, in basal metazoans, it is unknown whether inheritance of $\mathrm{CpG}$ methylation patterns occurs across the genome (as in plants) or as rare exceptions (as in mammals) ${ }^{4}$. Here, we demonstrate genome-wide intergenerational transmission of $\mathrm{CpG}$ methylation patterns from parents to sperm and larvae in a reef-building coral. We also show variation in hypermethylated genes in corals from distinct environments, indicative of responses to variations in temperature and salinity. These findings support a role of DNA methylation in the transgenerational inheritance of traits in corals, which may extend to enhancing their capacity to adapt to climate change.

Corals provide habitat for thousands of marine species, protect shorelines and support human livelihoods ${ }^{5}$. However, as the oceans warm, repeated thermal stress events have driven corals into severe global decline ${ }^{6,7}$, and the rapid rate of climate change threatens to overwhelm the capacity for adaptation by genetic means alone ${ }^{8,9}$. Recent research has demonstrated that stress-induced changes in the DNA methylome of corals correlates with phenotypic changes that explain increased organismal fitness ${ }^{10,11}$. Mechanistically, these changes appear to regulate transcriptional homeostasis through the inhibition of spurious transcription and transcriptional noise, reflecting adjustments of expression in response to transcriptional needs under changing conditions ${ }^{10,12}$. However, due to the low heritability of DNA methylation in mammals and its complete absence in popular model organisms (e.g., Drosophila and 
39 contribute to transgenerational plasticity, and hence to the ability of corals to respond to

$40 \quad$ climate change ${ }^{9}$.

41 Here, we initiate a paradigm shift by providing evidence for intergenerational inheritance of

42 DNA methylation patterns in reef-building corals. The brain coral Platygyra daedalea ${ }^{13}$ has

43 life-history traits which we expect to promote epigenetic acclimatisation within generations,

44 and the transfer of these modifications between generations ${ }^{8}$. Namely, individual colonies

45 are typically stress-tolerant ${ }^{14}$, long lived (up to $\sim 100$ years, Supplementary Table S1), and

46 spawn large numbers of gametes annually ${ }^{15}$. Samples of $P$. daedalea were obtained from two

47 populations in the Arabian Peninsula (Fig. 1a, b). The Abu Dhabi population $\left(24^{\circ} 35^{\prime}\right.$ '56" N,

$4854^{\circ} 25^{\prime} 17^{\prime \prime} \mathrm{E}$ ) is highly stress-tolerant, lives under extreme temperatures (winter $<19^{\circ} \mathrm{C}$ and

49 summer $>35^{\circ} \mathrm{C}$ ) and salinity (40-46 psu; Supplementary Table S2), and has persisted

50 through major thermal stress events (coral bleaching) that occurred in Abu Dhabi in 1998,

512002,2010 , and $2012^{16,17}$. In contrast, the neighbouring Fujairah population $\left(25^{\circ} 29^{\prime} 33^{\prime}\right.$ N ,

$\left.5256^{\circ} 21^{\prime} 50^{\prime \prime} \mathrm{E}\right)$ lives under comparatively milder temperatures $\left(22-33^{\circ} \mathrm{C}\right)$ with near-oceanic

53 salinity (36-39 psu) and has not experienced anomalous temperature events in recent years. 
a

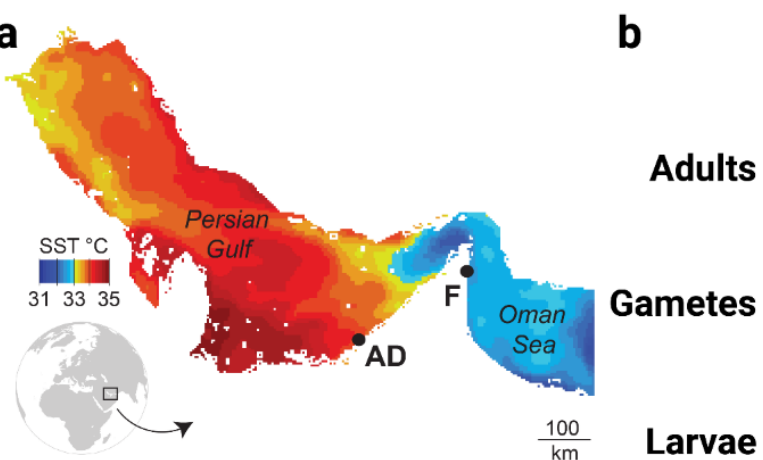

C $\begin{aligned} & 0.7 \\ & 0.6 \\ & 0.5 \\ & 0.4\end{aligned}$

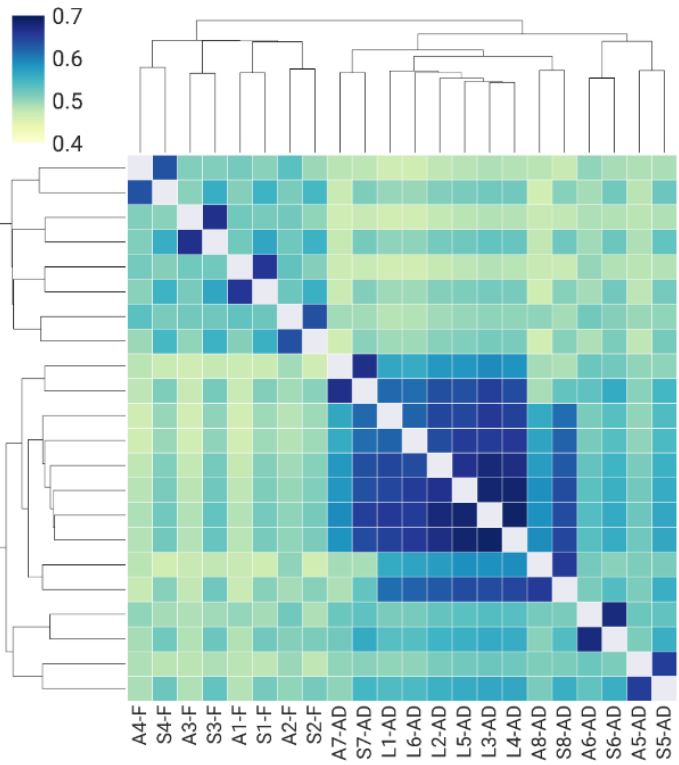

Fujairah (F)
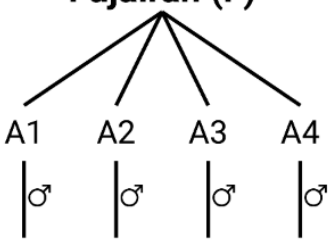

S1 $\quad$ S2 $\quad$ S3 $\quad$ S4
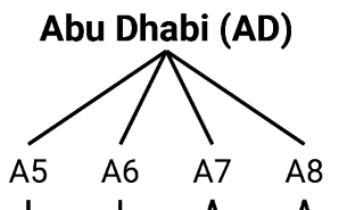

lon

S5 $\quad$ S6 $\quad$ E7 S7 E8 S8

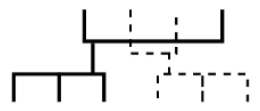

L1 L2 L3 L4 L5 L6

d

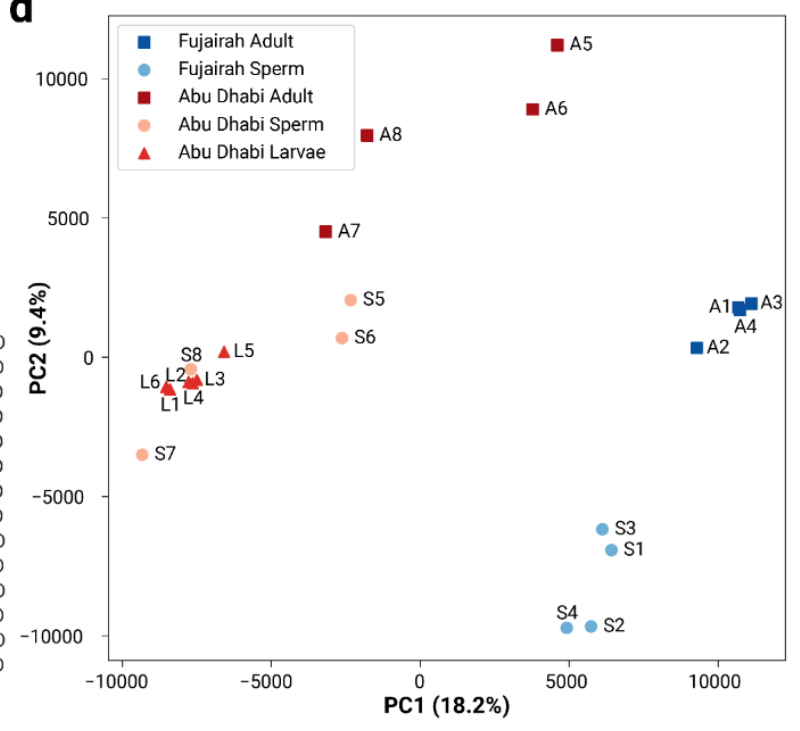

Fig. 1: Data acquisition and summarised findings showing intergenerational inheritance

of DNA methylation patterns in P. daedalea. (a) Contour map showing summer sea surface temperature (SST) differences between origin populations at Fujairah (F) and Abu Dhabi (AD) (mean of $5 \mathrm{~km}$ night-time SST for August 2015, NOAA, https://coralreefwatch.noaa.gov/satellite/bleaching5km/index.php). (b) Samples were collected from eight adult colonies (A1-A8) from Fujairah (A1-A4) and Abu Dhabi (A5A8). Sperm (S1-S8) and eggs (E7, E8) were collected from the correspondingly numbered adult. Larval samples were produced from reciprocal crosses: three from E7 and S8 (L1-L3), and another three from S7 and E8 (L4-L6). Data from egg samples (E7 and E8) were excluded from downstream analyses due to poor qualities and insufficient coverages. (c) Clustering was performed on the pairwise correlation of methylation data from all samples. 
samples with their respective adults, and the larval samples with samples from their parents direction in which crosses were performed. Additionally, samples clustered well by geographical origin (“-F”: Fujairah, “-AD”: Abu Dhabi). Colour bar shows Kendall rank correlation coefficient $(\tau)$. (d) A PCA based on the same methylated positions exhibits clear separation of samples based on geographical origin (blue: Fujairah, red: Abu Dhabi) along PC1, and by developmental stage (squares: adults, circles: sperm, triangles: larva) along PC2.

76 Using whole genome bisulphite sequencing (WGBS) data from 30 Illumina HiSeq2000 lanes, we identified 1.73 million $\mathrm{CpG}$ positions that were consistently methylated in the $\sim 800 \mathrm{Mb} P$. daedalea genome (3.91\% of all CpGs). These positions had a per-sample mean coverage of 45.44× across 22 samples (Supplementary Data S1b). Similar to other studied cnidarians, 80 methylation in P. daedalea was predominantly in gene bodies ${ }^{10-12,18,19}$. A larger fraction of

$81 \mathrm{CpGs}$ in genic regions were methylated than in intergenic regions (5.0\% vs. $3.6 \%$ respectively; Supplementary Fig. S1a). On a per-position basis, methylated positions in genic regions were also, on average, more strongly methylated than in intergenic regions (mean of $34.2 \%$ vs. $23.2 \%$ respectively; Supplementary Fig. S1b). Within genic regions, methylated positions were more commonly found at both ends (Supplementary Fig. S1c). 
a

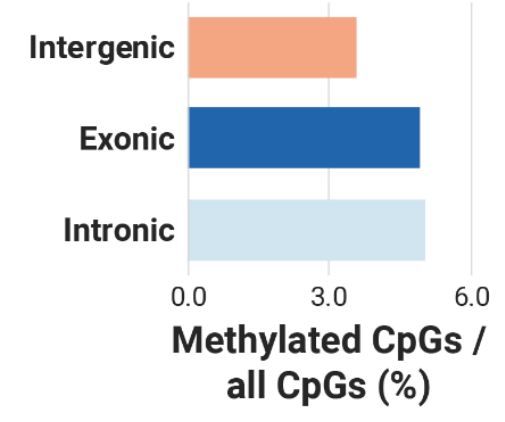

C

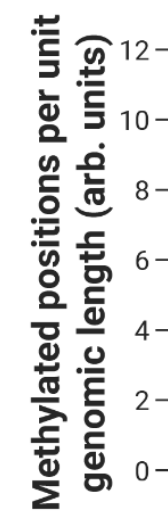

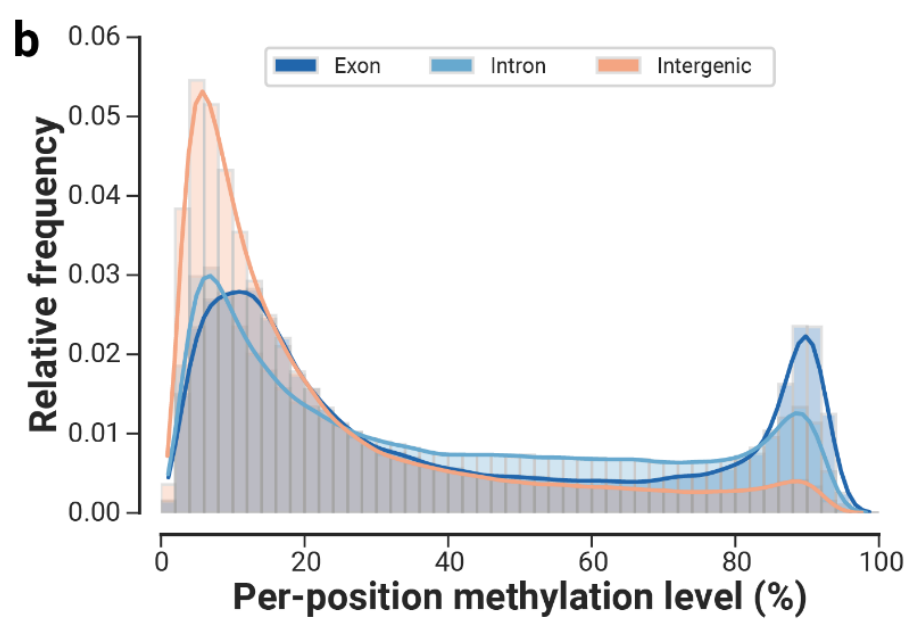

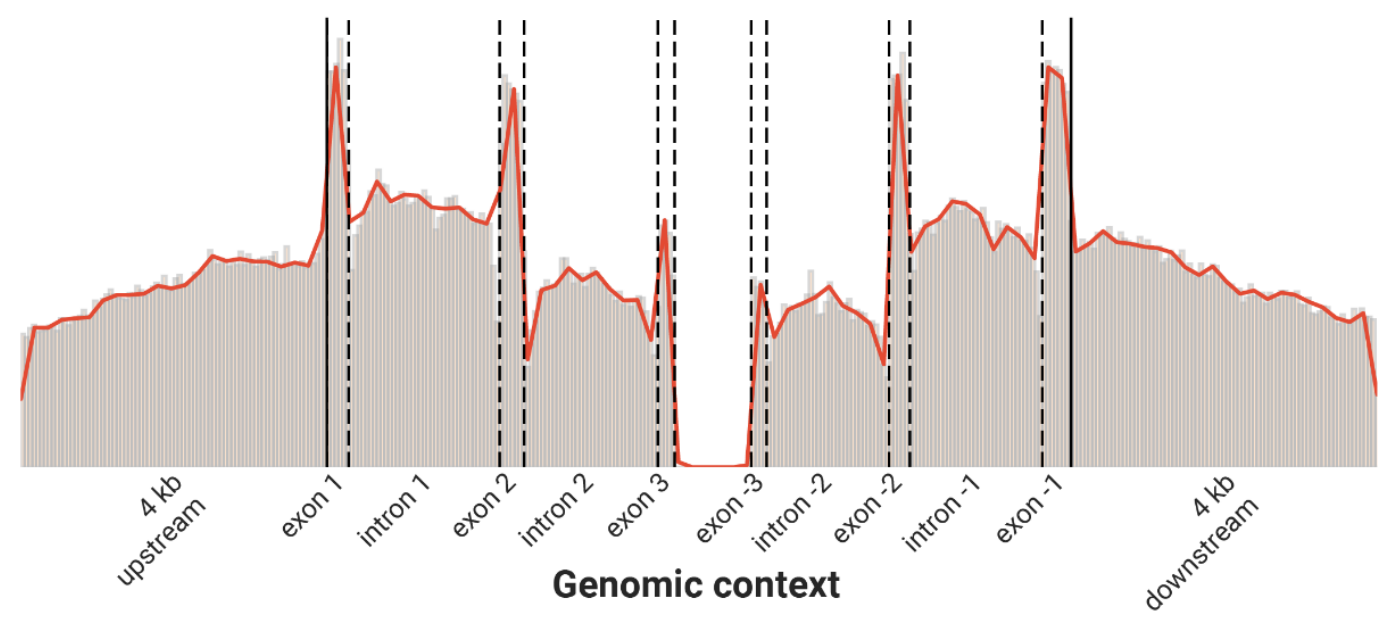

Supplementary Fig. S1: Methylation in P. daedalea is more commonly found in genic regions, and concentrated closer to the 5' and 3' ends. (a) Genic regions are more frequently methylated ( $5.0 \%$ CpGs are methylated) than intergenic region (3.6\%). (b) Methylation levels are bimodally distributed in exons, introns and intergenic regions. Exons have the highest methylation levels, followed by introns and intergenic regions. (c) Relative frequencies of methylated positions across a standardised gene model with flanking $4 \mathrm{~kb}$ regions indicate that methylated positions are more frequently found at both ends of the gene model. Solid lines depict transcriptional start site (left) and transcription termination site (right) while dotted lines delineate the borders of the indicated genomic feature. The widths of the features correspond to mean normalised lengths of the respective exons and introns in P. daedalea (exons, from left to right: 286 bp, 320 bp, 225 bp, 203 bp, 270 bp, 380 bp; introns, from left to right: $1,971 \mathrm{bp}, 1,744 \mathrm{bp}, 1,598 \mathrm{bp}, 1,728 \mathrm{bp}$ ). 
101 Parental genotype proved to be a strong predictor of inherited P. daedalea DNA methylation

102 patterns: sperm samples were significantly more similar to their respective parents than non-

103 parental sperm (mean $\tau \pm$ SEM: $65.7 \pm 0.2 \%$ vs. $53.2 \pm 0.1 \%, t$-test $p<10^{-16}$; Fig. $1 \mathrm{c}$ ). This

104 signature of inheritance was also present in the larval samples - as a group, they were

105 significantly more similar to their parental sperm samples (S7 and S8) than to other sperm

106 samples (S1-6; $60.5 \pm 0.1 \%$ vs. $51.0 \% \pm 0.1 \%, t$-test $\left.p<10^{-24}\right)$. Within the group, however,

107 the larval samples did not cluster in a pattern that reflected the reciprocal crossings,

108 suggesting the crosses exhibit equal paternal and maternal effects. Remarkably, the variation

109 of the methylation data captured by the first two principal components in a PCA (principal

110 component analysis) shows a clear separation of the samples by geographical origin along

111 PC1 (Fujairah/Abu Dhabi), and by developmental stage along PC2 (adult/sperm/larval; Fig.

112 1d). While the effect of genotype is not apparent in the first two principal components, PCAs

113 of samples plotted per-location (i.e. excluding effects of geographical origin) show clustering

114 of samples by genotype (Supplementary Fig. S2). In all PCAs, larval samples clustered

115 closest to their parental sperm samples, and in a gender-neutral fashion. 

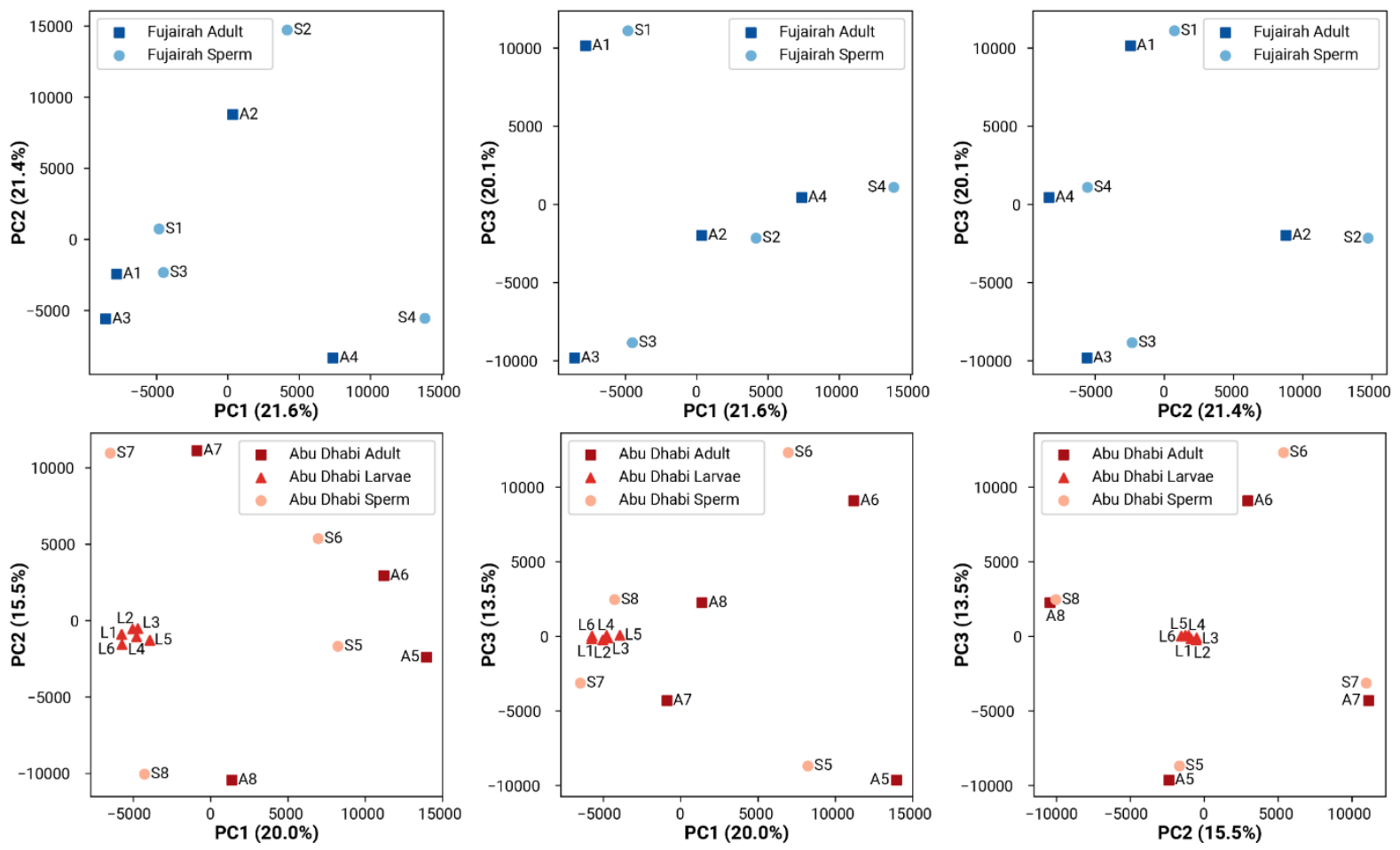

118 Supplementary Fig. S2: Per-location PCAs of DNA methylation patterns in P. daedalea.

119 PCAs were carried out on Fujairah (top row) and Abu Dhabi samples (bottom row) separately

120 on the first three principal axes. Samples tended to pair by genotype. The larval samples (red

121 triangles) are positioned approximately midway of sperm samples S7 and S8 along all plotted

122 axes, suggesting equal contribution from both parents to their DNA methylation patterns.

124 As we were not dealing with genotypically identical samples, we wondered - to what

125 extent - the observed epigenotypes of $P$. daedalea were determined by their individual

126 genotypes. If epigenotypes were perfectly correlated to genotypes, it implies that the

environment could only influence epigenotype via natural selection of better adapted

128 genotypes. To estimate variation in the genotype of our samples, we analysed the WGBS

129 reads and identified 407k SNPs present in the A/T fraction of the genome (as A and T bases

130 remain unchanged post-bisulphite treatment). Pairwise correlations of the genetic variation

131 were as expected: sperm samples were most similar to their respective parents, and there 
were fewer genetic differences between samples from the same location (Fig. 2a). If genotypes fully dictated epigenotypes, it follows that there would be a perfectly linear

134 relationship between pairwise genotypic correlations and epigenotypic correlations. Overall,

135 there is a strong but imperfect linear relationship $\left(r^{2}=0.805, p<10^{-43}\right)$ between these

136 variables (Fig. 2b). Pairwise comparisons with the highest genetic correlations (i.e. adult vs.

137 sperm of the same genotype) had the highest epigenetic correlation. When comparing samples across locations (Fujairah vs. Abu Dhabi, purple), they were genetically—and epigenetically_less similar than samples from the same location (blue and red), illustrating

140 the combined effect of genotype and environment on epigenotypes. Focusing on the vertical

141 spread of epigenetic correlation values, comparisons of adult vs. sperm samples (intermediate

142 hues) result in correlations with lower values than adult vs. adult (dark hues) or sperm vs. sperm (light hues). This indicates that development has an effect on epigenotype.

144 To further deconvolute the separate effects of genotype and the environment on the

145 epigenotype, we calculated $\mathrm{F}_{\mathrm{ST}}$ values of genomic loci in $50 \mathrm{~kb}$ windows to identify regions

146 that are genetically homogeneous/heterogeneous between the populations (Fujairah vs. Abu

147 Dhabi). If genotype fully influences epigenotype, in genetically homogeneous loci, we expect

148 to see approximately equal amounts of methylation in both populations (i.e. delta methylation

$149=0$ when $\mathrm{F}_{\mathrm{ST}}=0$ ); in genetically heterogeneous loci, we expect to see large differences in

150 methylation levels between populations. Surprisingly, homogeneous loci have a median delta

151 methylation of $1.2 \%$ (IQR: $-0.7-3.4 \%$ ), which significantly departs from expectations (one

152 sample $t$-test $p<10^{-132}$ ). As expected, we also observe progressively larger differences in

153 methylation levels in more heterogeneous loci (Fig. 2c). This finding demonstrates that a

154 strong environmental effect contributes to the epigenotypes of $P$. daedalea. 


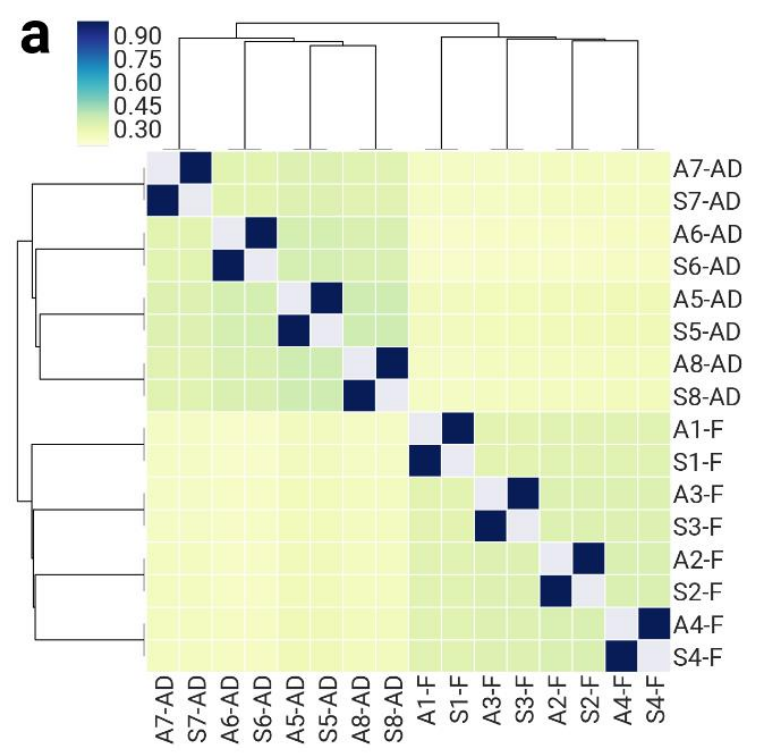

b
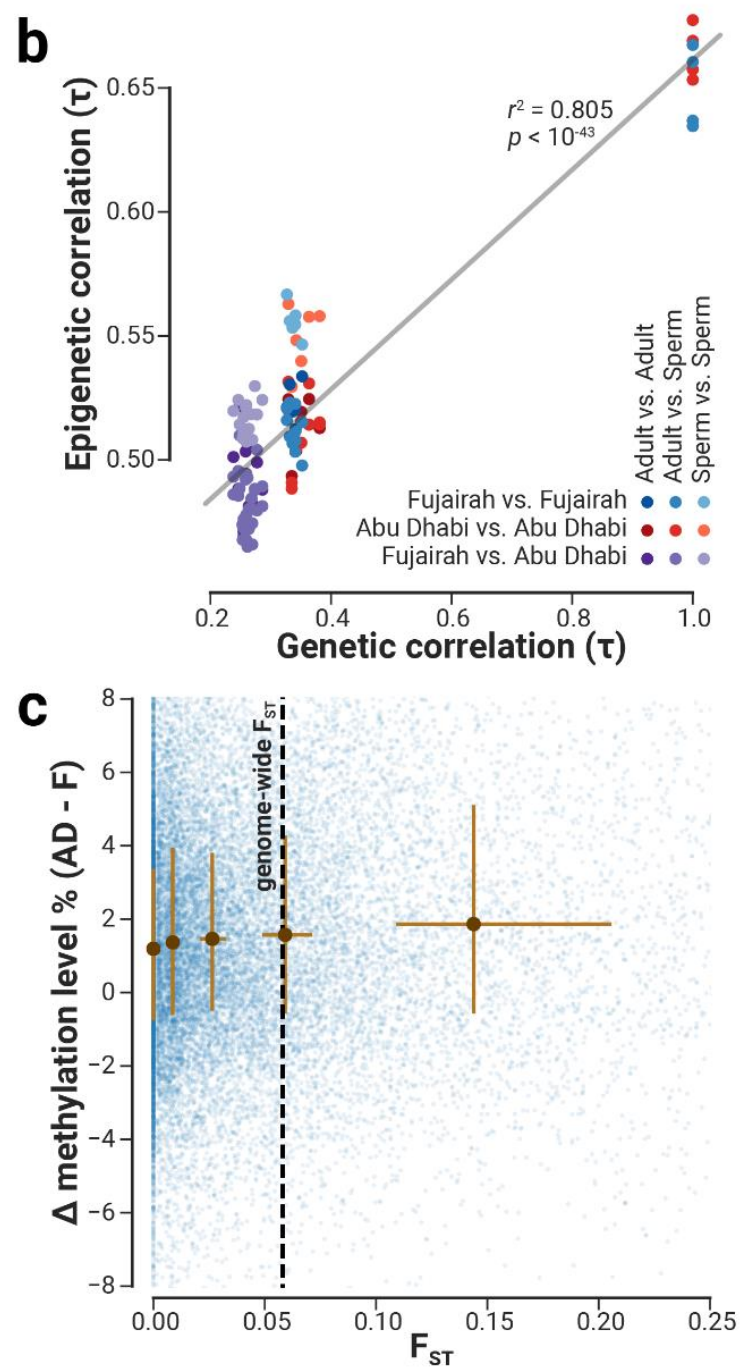

157 Fig. 2: Epigenotype is strongly influenced by genotype, and to a lesser extent, by

158 environment and development. (a) Clustering was performed on the pairwise correlation of 
SNP data from adult and sperm samples $(n=16)$. Samples cluster best by individual, followed by the geographical origin of the samples. Colour bar indicates Kendall rank

162 comparison (i.e. 16 samples: ${ }^{16} \mathrm{C}_{2}$ possible pairings $=120$ points), the pairwise genetic

163 correlation $(\tau)$ is plotted on the $\mathrm{x}$-axis (Supplementary Data $\mathrm{S} 2 \mathrm{~b}$ ), while the pairwise

164 epigenetic correlation $(\tau)$ is on the y-axis (Supplementary Data S2c). Points were coloured according to sample identities in the pairwise comparison (see bottom-right colour matrix).

167 purple otherwise); hues are assigned based on developmental stage (darkest hue if both are

168 adults, lightest if both sperm, intermediate otherwise). Pairwise genetic similarities are

169 strongly, but not completely, predictive of epigenetic similarities. The combined effects of genotype and environment contribute to the horizontal spread of points; developmental stage contributes to the vertical spread of points. (c) $\mathrm{F}_{\mathrm{STS}}$ and differences in median methylation levels (Abu Dhabi - Fujairah) between populations were calculated for all genomic loci (50 kb window, $10 \mathrm{~kb}$ step size) in the $P$. daedalea genome. Window-specific delta methylations were plotted for windows with $\geq 60$ methylated positions and $\geq 20$ SNPs. As FST values were not normally distributed, they were partitioned into quintiles. Brown dots indicate median FSTS and median methylation differences for every quintile; brown lines indicate interquartile ranges.

We proceeded to investigate whether developmental and environmental effects had a combined effect on the epigenotypes of $P$. daedalea. Using a generalised linear model

181 (GLM), we confirmed that genic methylation levels $(n=11,598$ genes with $\geq 5$ methylated

182 positions) were almost always $(n=11,594)$ independently affected by development and environment, supporting the independent analysis of these effects. 
184 We were interested in the functional significance of genes that undergo large changes in

185 methylation levels between developmental stages. We observed generally higher methylation

186 levels in sperm samples than their parents (median: $+3.2 \%$, mean: $+5.2 \%$; Fig. $3 \mathrm{a}$ ), and these

187 increases are mainly located in genic regions (Fig. 3b). As invertebrate DNA methylation is

188 concentrated in gene bodies ${ }^{10,12,20-22}$, the targeted increases in methylation levels are likely to

189 have functional significance. To identify genes that are differentially methylated in a

190 development-specific manner, we carried out a one-way ANOVA and filtered by effect size

191 (corrected $p<0.05$ and methylation levels differ $>20 \%$ between any pair of developmental

192 stages) to produce a list of 794 genes (6.8\% of all methylated genes). 

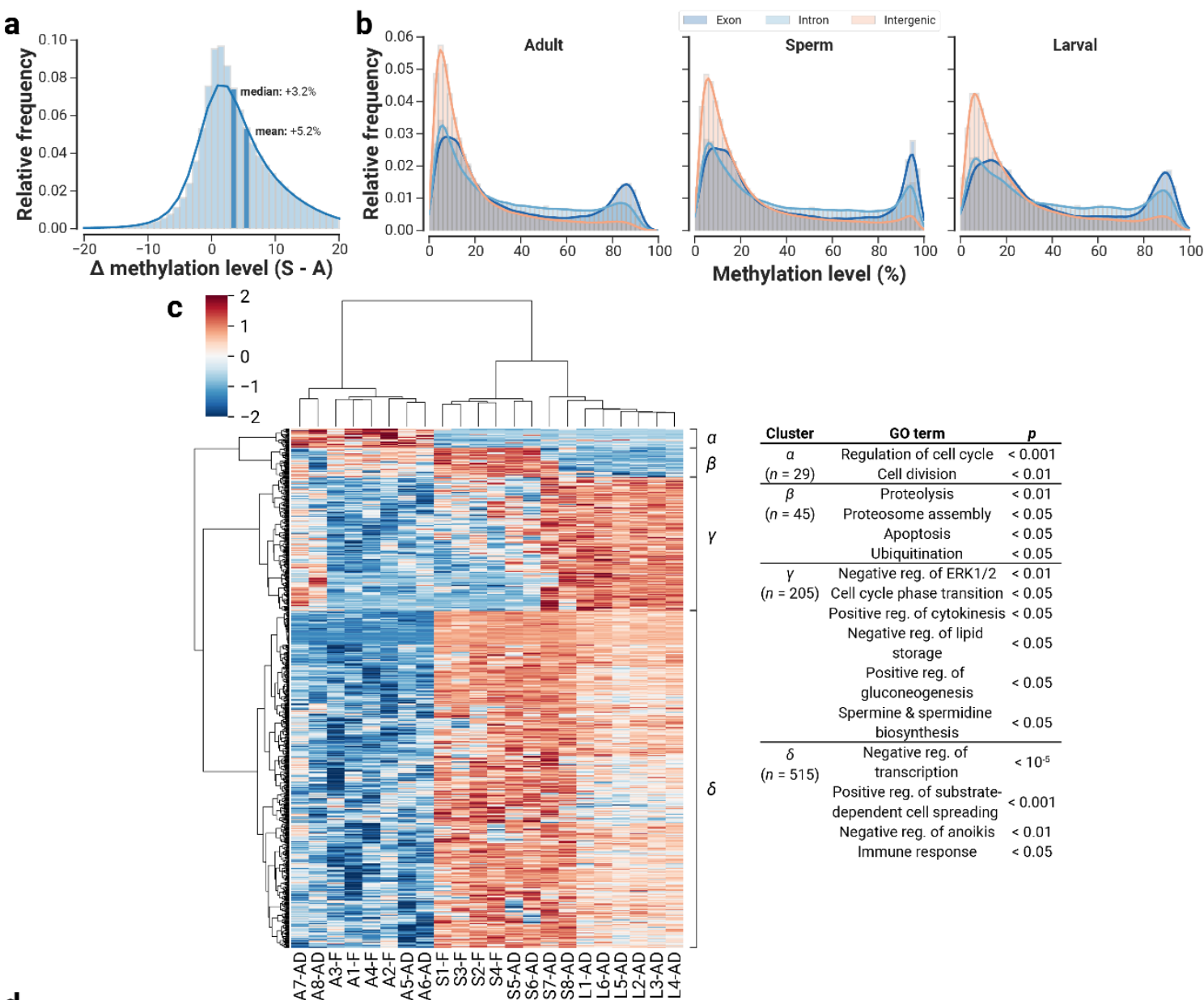

$(n=205)$ Cell cycle phase transition $<0.05$ Positive reg. of cytokinesis $<0.05$ Negative reg. of lipid $<0.05$ storage

Positive reg. of gluconeogenesis $<0.05$ permine \& spermidine

\begin{tabular}{ccc} 
& biosynthesis & $<0.05$ \\
\hline$\delta$ & Negative reg. of & $<10^{-5}$ \\
$(n=515)$ & transcription &
\end{tabular}

$(n=515)$ Positive reg. of substrate- $<0.001$

Negative reg an

$\begin{array}{ll}\text { Immune response } & <0.05\end{array}$

\begin{tabular}{|c|c|c|c|c|c|c|c|c|c|c|c|}
\hline a & 452, & & & & 5,000 & & & & & & 462,50 \\
\hline A1-F & & , & .1 & & & & 111 & $\left.{ }^{m}\right]$ & \|\|$\|$ & 1,1 & |||||| $\mid$ \\
\hline A2-F & & .1 & .1 & ". & & & .11 & $\left.{ }^{\infty}\right]$ & "1 & 111 & |||||| \\
\hline A3-F & & 11 & & ". & . & & II & ${ }^{\infty}{ }^{3}$ & \|\| & 111 & $\mid$ | || || \\
\hline A4-F & & & &. & 1. & & .11 & 呰国 & "il & 111 & | | || || \\
\hline A5-AD & & .1 & 11 & & & & $\ldots$ & 紧目 & $\|n\|$ & 111 & $11|1|$ \\
\hline A6-AD & & 1. & .1 & & & & $\ldots 1.1$ & wa & "11 |11 & 1,1 & $\mid$ | ||$||$, \\
\hline A7-AD & & 11 & .1 & $"$. & & & , 111 & 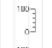 & \|\|$\|$ & 111 & |||||| \\
\hline A8-AD & & . 1 & , & & & & $\ldots$ & to & \|ा & 111 & |||||| \\
\hline S1-F & & 11 & | | & || | | & |||||| $\mid$ & | || & |||||| & m & .1.... & 1,1 & | | || || \\
\hline S2-F & & 11 & 11 & $\|\mid\|$ & |||||| $\mid$ & | || & |||||| & $m_{0}$ & |nil| & 111 & | | || || \\
\hline S3-F & & 11 & 11 & $\|||$ & ||||$,|| \mid$ & | || & |||||| & "'m & \|\|$\|$ & 111 & | | ||| || \\
\hline S4-F & & | & | & $\|||$ & | | || || & | || & |||| $\mid$ & m & \|\|$\|$ & 111 & | | || || \\
\hline S5-AD & & | | & | | & $\|||$ & & & $|\||||$ & m & \|\|$\|$ & 111 & $|1|||$ \\
\hline S6-AD & & 11 & & $\| \mid$ & & & |||| $\mid$ & ; & nin & 1,1 & |||||| \\
\hline S7-AD & & || & | | & $\| \mid$ & & & |||||| & $m$ & inin & 111 & |||||| \\
\hline S8-AD & & 11 & & $\|||$ & & & |||||| & "io & init & 111 & $|1|||$ \\
\hline L1-AD & & 11 & 11 & $\|||$ & ||$\||| \mid$ & | || & | || ||| & " & \|\|$\|$ & 111 & |||||| \\
\hline L2-AD & & 11 & 11 & $\| \mid$ | & | | || ||| & | || & || $\mid \|_{1}$ & 奌: & \|\|$_{1}$ & $1 \mid 1$ & |||||| \\
\hline L3-AD & 1 & 11 & 11 & $\| \mid$ | & |||||| $\mid$ & | || & |||||| & ${ }^{100}:$ & \|\|$\|$ & 111 & | 1|||| \\
\hline L4-AD & , & 11 & 11 & $\| \mid$ | & |||||| $\mid$ & I l| & | || ||| & ${ }^{w}$ & \|\|$\|$ & 111 & | | || || \\
\hline L5-AD & & 11 & .1 & $\|$ | & | | || ||| & I $\|$ & | || ||| & 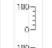 & \|\|$\|$ & 111 & | 1 || || \\
\hline L6-AD & & | 11 & 11 & $\| I \mid$ & |||||| $\mid$ & | || & | || ||| & ${ }^{10}$ 晋 & \|\|$\|$ & 111 & |||||| $\mid$ \\
\hline
\end{tabular}

Fig. 3: DNA methylation profiles vary considerably across developmental stages. (a)

196 There is a general increase in per-position methylation levels in the sperm samples, relative to 
their parents. (b) This increase is more pronounced in genic regions (i.e. exon and introns) than intergenic regions. (c) Clustering of differentially methylated genes recapitulates the samples are closest to their parents. Clustered genes were partitioned into four distinct groups (labelled as $\alpha-\delta$ ) for functional enrichment analysis: $\alpha, \beta$ and $\gamma$ represent genes that are hypermethylated in adults, sperm and larvae respectively; while $\delta$ represent genes that are hypermethylated in both sperm and larvae. Values of colour bar (-2 to 2) represent z-scores. (d) An example gene (Pdae13593, putative negative regulator of anoikis) that was consistently hypermethylated among the sperm and larval samples (S1-8, L1-8).

207 To investigate whether genes with similar biological functions share similar methylation 208 patterns across developmental stages, we performed a clustering analysis on the differentially 209 methylated genes. We observed four distinct clusters: genes that are hypermethylated for a 210 particular developmental stage and hypomethylated otherwise (clusters $\alpha, \beta, \gamma ;$ Fig. 3c), and 211 one that is hypermethylated in sperm and larvae but hypomethylated in adults (cluster $\delta$, Fig.

212 3c). Interestingly, methylation patterns in sperm samples tend to be the intermediate of adults 213 or larvae, suggesting that the altering of methylation levels during development is a gradual

214 process. Regardless, our data indicates that changes in methylation could occur in the

215 timescale of days (sperm and larval samples are 54 hours apart), in contrast to previously 216 published studies which measured changes in samples conditioned over weeks to years (6

217 weeks for Putnam, et al. ${ }^{23} ; 3$ months for Dixon, et al. ${ }^{11} ; 2$ years for Liew, et al. ${ }^{10}$ ).

218 Functional enrichment on individual clusters resulted in terms associated with the general

219 biological requirements of each stage. Hypermethylated genes in adults (cluster $\alpha$, Fig. 3c) 
of cell division in adults than gametes or larval samples. The preponderance of cell lysis and

224 spermatogenesis by regulating biogenesis and stability of membranous organelles ${ }^{24}$ and preventing inheritance of male mitochondria ${ }^{25}$. For hypermethylated genes in larvae (cluster $\gamma$, Fig. 3c), the terms indicate a pro-growth phenotype. The breakdown of fatty acids, gluconeogenesis, and the synthesis of spermine and spermidine suggest increased metabolic activity, while the regulation of cell cycle and ERK proteins, a family of kinases involved in and larvae (cluster $\delta$, Fig. 3c) appear to be linked to substrate recognition (Fig. 3d shows an example gene) and symbiont recognition, both important processes that underpin successful larval settlement and symbiosis establishment ${ }^{27,28}$.

In light of recent evidence for methylation changes conferring stress acclimatisation in corals 10,11, we were particularly interested in exploring the effect of environment on methylation levels. A similar approach with similar filters (corrected $t$-test $p<0.05$ and pairwise methylation levels differ > 10\%) identified 329 genes that were differentially methylated

237 between Fujairah and Abu Dhabi. The choice of a lower effect size filter stems from the 238 observation that methylation levels of samples from Abu Dhabi were not much higher than

239 from Fujairah (median: $+0.8 \%$, mean: $+1.7 \%$; Fig. $4 a$ ). This increase was also not specific to genic regions, unlike the hypermethylation observed in sperm relative to adults (Fig. 4b). 

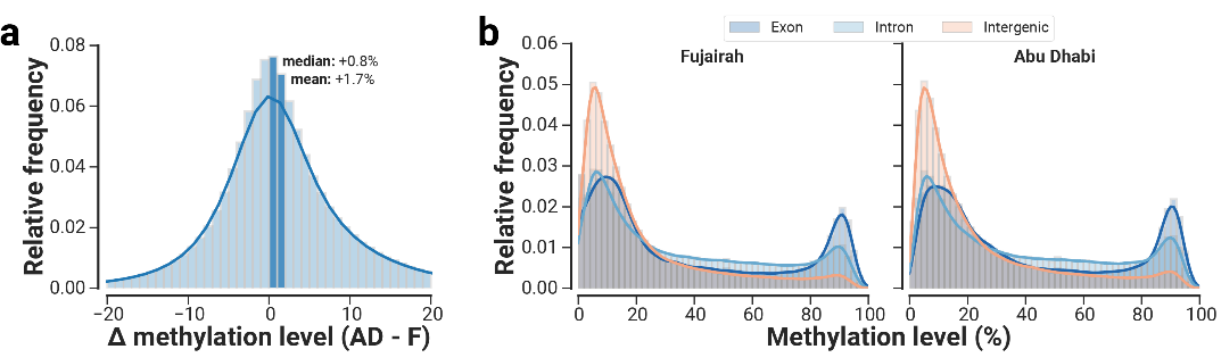

C
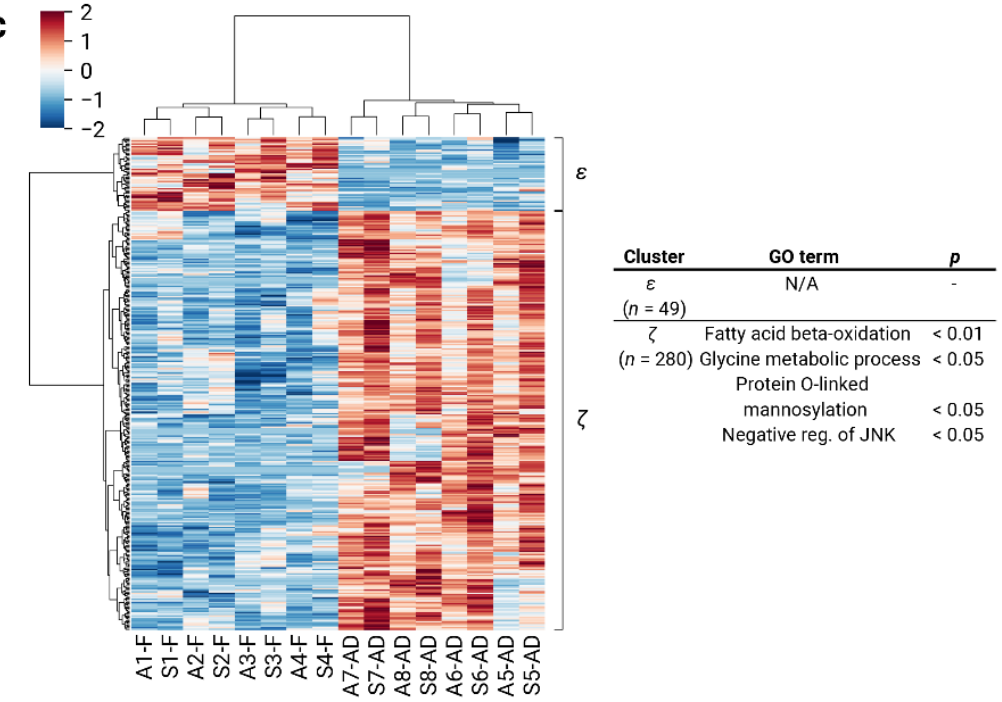
621,250

622,500

623,750

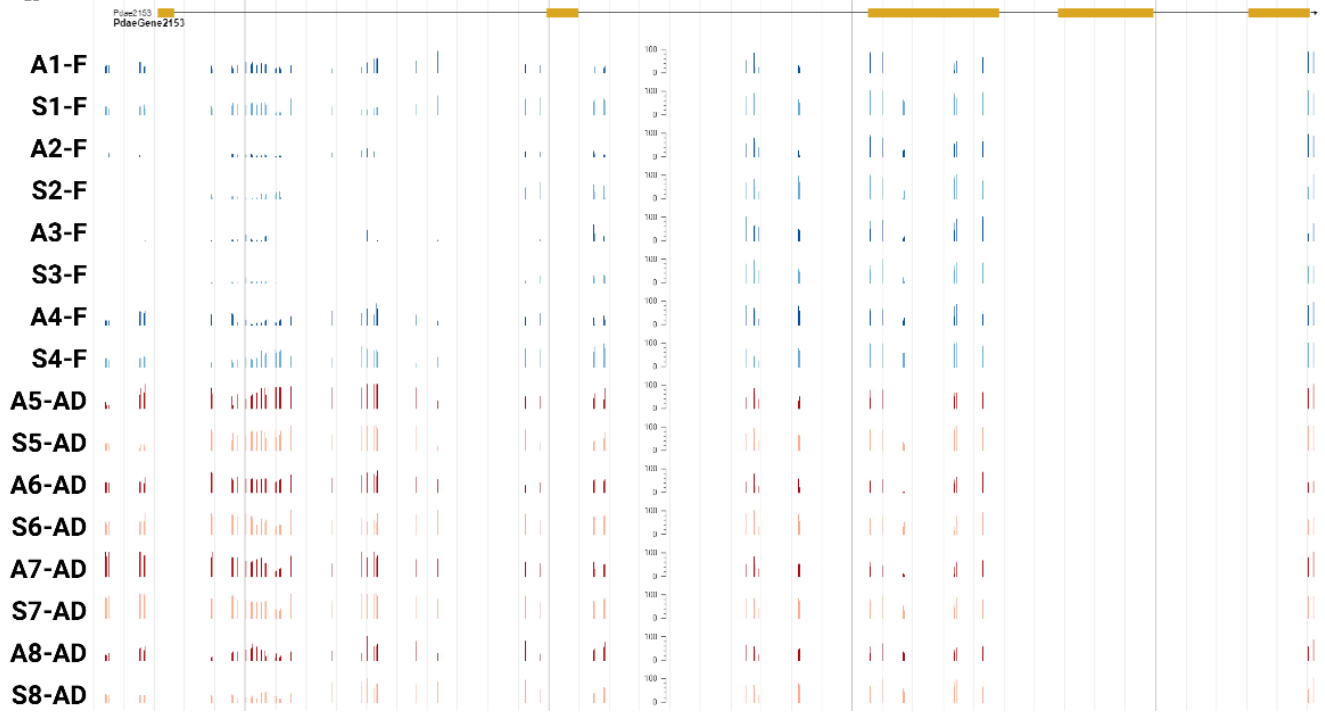

243 Fig. 4: DNA methylation profiles are also associated with the environmental origin of

244 the samples. (a) Samples from Abu Dhabi have slightly higher per-position methylation

245 levels than those from Fujairah. (b) This increase is not confined to a specific genomic

246 context. (c) There were fewer genes consistently hypermethylated among the Fujairah

247 samples (cluster $\varepsilon$ ) than in the Abu Dhabi samples (cluster $\zeta$ ). Notably, sperm samples

248 clustered best with their corresponding parent, further corroborating the intergenerational 
inheritance of methylation patterns. Values of colour bar (-2 to 2) represent z-scores. (d) An also appears influenced by genotype. to be responses to the hotter and more saline conditions of Abu Dhabi. Increased metabolism of fatty acids under heat stress is common in corals ${ }^{29,30}$ and sea anemones ${ }^{31}$. Glycine, in the form of mycosporine-glycine, is one of the primary mycosporine-like amino acids that confer photoprotective and antioxidative function in corals ${ }^{32}$. In yeast, O-linked mannosylation has

been shown to mark misfolded proteins that arise under cell stress, targeting them for

260 degradation in the endoplasmic reticulum ${ }^{33}$. The hypermethylation of JNK (c-Jun N-terminal

261 kinase) regulators (Fig. 4d shows an example gene) echoes similar observations in the coral

262 Stylophora pistillata: samples under long-term $\mathrm{pH}$ stress exhibited hypermethylation in the

JNK pathway that were strongly predictive of the resulting phenotype ${ }^{10}$. For the

functions as many of those genes had no functional annotations.

267 In conclusion, we demonstrate - for the first time - that epigenetic modifications in reef-

268 building corals are readily transmitted from parents to their offspring. This mode of

269 epigenetic inheritance is similar to that of plants, instead of other well-studied metazoans

270 (Supplementary Fig. S3). There are two possible explanations, keeping in mind conflicting

271 molecular evidence for the existence of segregated germlines in corals ${ }^{34-36}$. Firstly, the

272 absence of a segregated germline in corals would allow for the passing down of somatic cell 
methylomes, akin to epigenetic inheritance in plants ${ }^{2}$. For example, in Arabidopsis thaliana,

274 transfer of somatic cell methylomes facilitates the retention of stress tolerance in offspring

275 following parental stress acclimation ${ }^{37}$. Alternatively, coral methylomes could escape

276 reprogramming in the presence of a segregated germline in a similar fashion to zebrafish

277 paternal methylomes ${ }^{38,39}$. Transfer of paternal methylomes plays an important role in

278 zebrafish embryogenesis, for example in guiding left-right asymmetry of organ positioning ${ }^{40}$.

279 Importantly, in corals, inheritance of functional DNA methylation could enable acclimatory

280 gains in stress tolerance accumulated during long colony lifespans to be transferred to large

281 numbers of gametes or larvae (i.e. transgenerational plasticity). These "epialleles" ${ }^{41}$ could

282 thus serve as a non-genetic substrate for Darwinian selection to act upon, and potentially

283 accelerate evolution ${ }^{42}$. By extension, long-term cultivation of corals under elevated

284 temperatures may be a viable approach for producing fitter epialleles that could be later

285 crossed into wild populations to enhance their resilience to rapid climate warming ${ }^{43}$. Critical

286 to these optimistic predictions is verifying that inherited epialleles do confer functional

287 advantages to corals across generations, which could be the focus of future investigations.

Germline segregation \& reprogramming (à la mice)

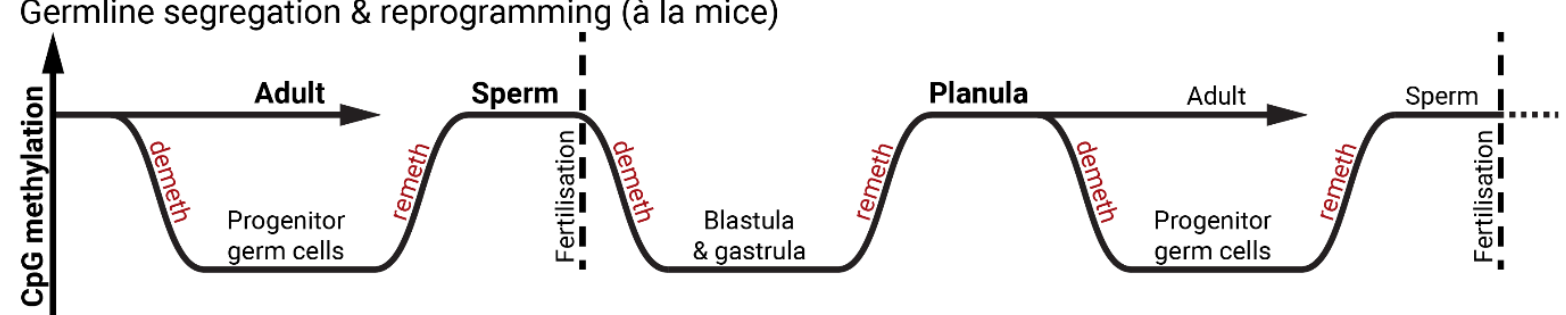

No germline segregation (à la zebrafish, plants)

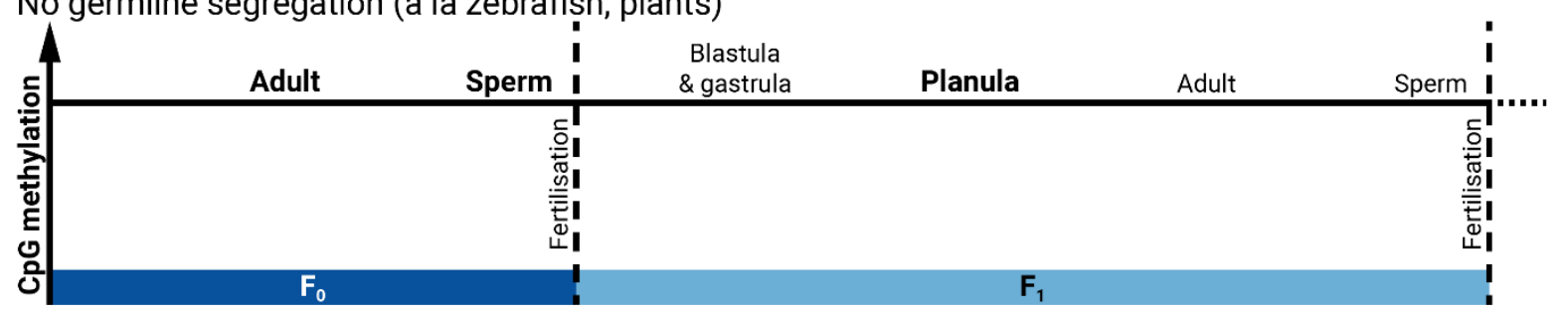




\section{Supplementary Fig. S3: Transgenerational inheritance of CpG methylation in corals}

modelled after mammalian or plants systems. Bolded text ("adult", "sperm" and

"planula") indicates equivalent samples for which we have methylation data in reef-building

corals. Consistent with both models, methylation levels from all of our samples are relatively

high. However, epigenetic reprogramming (twice per generation) in mice prevents the transgenerational inheritance of most epigenetic variation acquired in adults; on the other hand, CpG methylation are not reprogrammed in plants.

Methods

\section{Sample collection}

300

$P$. daedalea colony fragments were sourced from reef sites in the Sea of Oman in the Indian

301 Ocean (Fujairah: 25²9’33” N, 56 21'50” E; 3 m) and the southern Persian Gulf (Abu

302 Dhabi: $24^{\circ} 35^{\prime} 56^{\prime \prime} \mathrm{N}, 54^{\circ} 25^{\prime} 17^{\prime \prime} \mathrm{E}$; $\sim 6 \mathrm{~m}$ ) which have distinct thermal and salinity profiles

303 (Supplementary Table S2). Fragments were collected 4-6 nights prior to the full moon on 4

304 May 2015 and housed in 1,000-litre recirculating aquarium systems at New York University

305 Abu Dhabi at the ambient temperature and salinity measured at the time of collection (F: and broadcast spawning activity was observed from -4 nights to +10 nights relative to the full moon, with most individual fragments releasing gametes over several nights ${ }^{44}$. During this period, samples were collected from adults (F: 4; AD: 4), sperm (F: 4; AD: 4), eggs (AD: 2), and larval crosses (AD: 2), snap-frozen and stored at $-80^{\circ} \mathrm{C}$. Prior to spawning, individual

311 coral fragments were isolated in buckets to prevent cross-contamination and fertilization.

312 Gamete bundles (eggs + sperm) were collected with transfer pipettes, sperm was isolated by

313 filtration (60 $\mu \mathrm{m}$ plankton mesh) and concentrated by centrifugation. Larvae were bred from reciprocal crosses of gametes from two Abu Dhabi colonies, reared in $1 \mu \mathrm{m}$ filtered seawater 
in triplicate cultures, and were sampled at 54 hours post-fertilization (i.e., planula stage of

\section{Preparation and sequencing of whole genome bisulphite libraries}

319 DNA were extracted from all samples by suspending them in $750 \mu$ of buffer $(100 \mathrm{mM}$ sperm and $20 \mu \mathrm{l}$ for eggs), and stored at $4{ }^{\circ} \mathrm{C}$. conversion with the EpiTect Bisulfite Kit (Qiagen, Hilden, Germany) was carried out with cycling conditions of $95^{\circ} \mathrm{C}$ for $5 \mathrm{~min}, 60^{\circ} \mathrm{C}$ for $25 \mathrm{~min}, 95^{\circ} \mathrm{C}$ for $5 \mathrm{~min}, 60{ }^{\circ} \mathrm{C}$ for $85 \mathrm{~min}$, $95^{\circ} \mathrm{C}$ for $5 \mathrm{~min}, 60^{\circ} \mathrm{C}$ for $175 \mathrm{~min}$, then 3 cycles of $95^{\circ} \mathrm{C}$ for $5 \mathrm{~min}, 60^{\circ} \mathrm{C}$ for $180 \mathrm{~min}$, and held at $20^{\circ} \mathrm{C}$ for $\leq 5$ hours. Subsequently, libraries were enriched with KAPA HiFi HotStart

332 Uracil+ ReadyMix PCR Mix (KAPA Biosystems, Wilmington, MA), following

333 manufacturer's instructions. Libraries were quality checked on a Bioanalyzer DNA 1K chip

334 (Agilent, Santa Clara, CA) and quantified using Qubit 2.0 (Thermo Fisher Scientific, Waltham, MA), prior to pooling in approximate equimolar ratios for sequencing.

336 Pooled libraries were initially sequenced on 24 lanes of HiSeq2000 (Illumina, San Diego,

337 CA). Based on the results of the sequencing, libraries with lower coverages were resequenced 
340 From both runs (i.e., 30 lanes), a total of 4.13 billion read pairs were obtained across 24

341 samples, and subsequently trimmed using cutadapt v1.8 ${ }^{45}$ (Supplementary Data S1a).

342 Following trimming, reads were mapped to the draft $P$. daedalea genome (REF),

343 deduplicated and scored on a per-position basis for methylated and unmethylated reads using

344 Bismark v0.17 ${ }^{46}$. At this stage, we excluded data from the egg samples $(n=2)$ from

345 subsequent analyses as the data from these samples were very poor (struck-out red rows in

346 Supplementary Data S1b).

347 To ensure that methylated positions were bona fide, four stringent filters were applied.

348 Firstly, on the pooled dataset, the probability of methylated positions arising from chance on

349 a per-position basis was calculated using a binomial distribution of $\mathrm{B}(n, p)$, where $n$

350 represents total coverage (methylated + unmethylated reads) and $p$ the probability of

351 sequencing error (set to 0.01 to mimic a Phred score of 20). Positions with $k$ methylated reads

352 were kept if $p(\mathrm{X} \geq k)<0.05$ (post-FDR correction). Secondly, positions were kept only if

353 there were at least a methylated read in all biological replicates of at least one biologically

354 meaningful group (i.e., $n=3$ or 4 Fujairah adults, Fujairah sperm, Abu Dhabi adults, Abu

355 Dhabi sperm or Abu Dhabi larvae). Thirdly, positions were retained only if median coverage

356 was $\geq 10$ and minimum coverage was $\geq 5$ across all 22 samples. These filtering steps were

357 very conservative: 20.6 million positions had at least 1 methylated read mapping to it, but

358 only 1.7 million positions passed all filters. The scripts used for this section (and further

359 theoretical justifications) are available at https://github.com/lyijin/working_with_dna_meth.

\section{Assignment of genomic context to methylated cytosines}

361 A Python script was used as previously described ${ }^{10}$. Briefly, the script reads the GFF3

362 annotations of the $P$. daedalea genome and the positional coordinates of the methylated

363 cytosines to assign genomic context to the methylated position. Distances to the 5' and 3' end 
364 of each genomic feature (gene/intergenic region/exon/intron) were calculated for downstream analyses.

366 Overall, 16,035 genes (64.2\% of all genes) had at least one assigned methylated position;

367 12,111 genes (48.5\%) had ten or more assigned methylated positions.

368

369

370

371

372

373

374

375

376

377

378

380

381

382

383

\section{Identification of SNPs in the P. daedalea genome}

Using the deduplicated BAM files produced from the Bismark pipeline (all adult and sperm samples, $n=16$ ), coverages across all bases were compiled into a table via samtools ("samtools mpileup", with the P. daedalea genome providing the per-position reference base). Reference bases corresponding to $\mathrm{C}$ and $\mathrm{G}$ were discarded, as bisulphite treatment converts C-to-T (and G-to-A on the opposite strand).

A Python script further imposed three rules to filter for potential SNPs that are highly covered: across all 16 samples, median coverage at that position must be $\geq 20$, and the minimum coverage $\geq 10$. The overall minor allele frequency (MAF), calculated by dividing the sum of all non-genome-matching bases over total coverage (i.e. values range from 0 to 1 inclusive), must be between 5\% and 95\%. A value below 5\% implies that the non-genomematching bases probably arose from sequencing errors; a value above $95 \%$ is likely due to the reference base being wrongly called. If a position passes these three criteria, MAFs were calculated on a per-sample basis and stored as an intermediate file.

Using another Python script, per-position genotypes were assigned based on the MAFs, as listed below:

MAF $\leq 25 \%$ : genotype 0 (i.e. homozygous reference base) $25 \%<$ MAF $\leq 75 \%$ : genotype 1 (i.e. heterozygous) MAF > 75\%: genotype 2 (i.e. homozygous non-reference base) 
387 As we assumed that the adult and sperm samples from the same individual should be

388 genetically identical, positions were only retained if all sperm samples and their respective

389 parents had the same genotype (i.e., A1-8, when written out as a list, would be identical to

390 S1-8 written out in a similar fashion). A total of 407,419 positions passed this final filter, and

391 were deemed SNPs.

\section{Calculation of Kendall ranked correlation coefficients for pairwise comparisons}

393 As both methylation data and SNP data were not normally distributed, we opted to calculate

394 the Kendall ranked correlation coefficients $(\tau)$ to measure whether data were similarly

395 ordered in a similar fashion in datasets $X$ and $Y$. The range of $\tau$ spans $[-1,1]$, where -1

396 indicates perfectly discordant ranked observations; 1 indicates perfectly concordant ranked

397 observations.

398 Correlation coefficients were calculated using Python scripts, which enabled the plotting of

399 Figs. 1c, 2a and 2b. For transparency, these values are tabulated in Supplementary Data S2.

\section{Assessing effect of genotype on epigenotype}

401 To assess the effect of genetic heterogeneity on DNA methylation levels, the $P$. daedalea

402 genome was partitioned into $50 \mathrm{~kb}$ windows with a step size of $10 \mathrm{~kb}$. Genetic heterogeneity

403 was estimated using $\mathrm{F}_{\mathrm{ST}}$ — but as there are various ways to calculate this value, we adopted

404 Hudson's definition and calculated per-position FST values using equation 10 described in

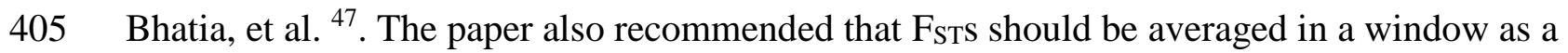

406 ratio of averages, using the equation (as derived in the Supplementary Materials of Bhatia, et

407 al. ${ }^{47}$ but reproduced here for convenience):

408

$$
\widehat{F}=\frac{\sum_{k=1}^{M} \widehat{N}^{[k]}}{\sum_{k=1}^{M} \widehat{D}^{[k]}}
$$


411 Hudson's equation respectively:

$$
\begin{gathered}
N=\left(p_{1}-p_{2}\right)^{2} \\
D=p_{1}\left(1-p_{2}\right)+p_{2}\left(1-p_{1}\right)
\end{gathered}
$$

414 where $p_{1}, p_{2}$ denote allelic frequencies of the non-reference base among the Fujairah and Abu

415 Dhabi populations respectively.

416 The epigenotypic variances within the same $50 \mathrm{~kb}$ windows were calculated by first

417 calculating the window-specific mean methylation levels across all Fujairah samples, and

418 again for all Abu Dhabi samples. The absolute difference between these two means thus

419 indicates the extent of the differences in methylation levels between the two populations.

420 As the draft genome still contains regions of unknown bases (Ns), we retained windows that

421 had $\geq 20$ SNPs and $\geq 60$ methylated sites. Correlations of these computed values were all 422 implemented in Python.

\section{Identification of differentially methylated genes}

424 Individual positions have methylation levels that range from 0 (not methylated at all in all

425 cells) to 1 (completely methylated in all cells). To extend this measure to genes, which

426 contain multiple methylated positions, medians of the methylation levels were calculated for

427 every gene (medians are less prone to outliers than means). Furthermore, to reduce noise, we

428 focused on genes that had at least five or more methylated positions, filtering out 3,068 genes

429 that did not meet this criterion. The genes that were filtered out were mostly unannotated, or

430 annotated as proteins with unknown functions. 
431 To ensure appropriate statistical models were applied for the analysis of our dataset, we first

432 checked whether genic methylation levels were normally distributed within biologically

433 meaningful groups ${ }^{48}$, and whether variances were equal between groups ${ }^{49}$. For the vast

434 majority of genes, the null assumptions of normality and equal variance could not be rejected

435 (Supplementary Data S3). Hence, we used untransformed methylation levels in all

436 subsequent statistical tests.

437 Genic methylation levels were thought to be influenced by two variables: developmental 438 stage (“devt": adult/sperm/larvae) and sample origin (“origin": Fujairah/Abu Dhabi). As the 439 interaction of both variables could confound statistical analysis that focused on individual 440 variables, a GLM (generalised linear model) was implemented in Python with the general 441 equation: methylation level $\sim$ devt + origin + devt:origin

443 Our results indicate that methylation levels of only 4 of 11,598 genes had a significant 444 interaction between developmental stage and origin (Supplementary Data S4). Consequently, 445 we infer that methylation level are independently affected by developmental stage and sample 446 origin, justifying the separate analysis outlined in Supplementary Fig. 4.

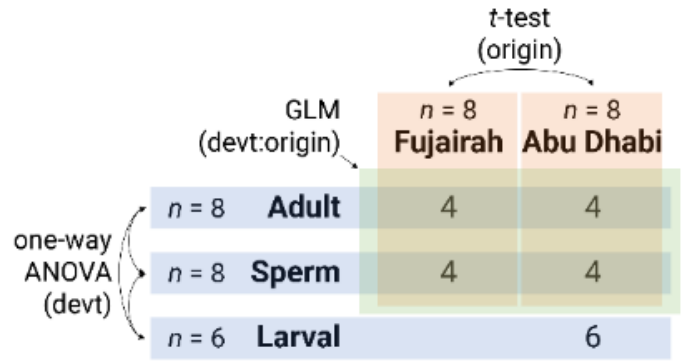

448 Supplementary Fig. 4: Outline of statistical tests performed. Numbers denote biological

449 replicates of different developmental stages and sample origins, while boxes denote the

450 groupings used in the statistical tests. The initial GLM (green) tested whether both variables

451 had significant interaction. Subsequently, the one-way ANOVA (blue) identified genes that 


\section{Multiple testing correction of $p$ values}

474 Unless otherwise stated, computed $p$ values were subjected to the Benjamini-Hochberg-

475 Yekutieli multiple testing correction ${ }^{50,51}$.

were differentially methylated across developmental stages, while the $t$-test (red) identified genes that were differentially methylated across sample origins.

To identify genes that were differentially methylated due to developmental stage, one-way ANOVAs were carried out on a per-gene basis encompassing three developmental stages $(n=$ 8 adults, 8 sperm, 6 larvae). As many genes were considered significant $(3,863$ of 11,598), we filtered for genes with a methylation level change of $>20 \%$ in any direction between any pair of developmental stages. This conservative filter picked out 794 genes that exhibited significant and large changes during development (Supplementary Data S5).

To identify genes that showed significant differences in methylation level due to geographical origin, $t$-tests were carried out on a per-gene basis for all Fujairah samples against non-larval Abu Dhabi samples ( $n=8$ Fujairah, 8 Abu Dhabi). Comparatively fewer genes (406 of 12,111) were considered significant. As samples from the different origins had similar methylation levels, a less stringent effect size filter of $>10 \%$ was applied, resulting in a final set of 329 genes that exhibited significant and large changes between locations (Supplementary Data S6).

Clustered heatmaps were plotted for these two classes of differentially methylated genes with seaborn (https://github.com/mwaskom/seaborn), a plotting library in Python. Non-default parameters include the choice of clustering method (Ward, instead of UPGMA), and the use of $\mathrm{z}$-score values (i.e. individual values reflect the number of standard deviations away from the mean). 


\section{Functional enrichments within genes of interest}

477 GO term annotations were obtained from the draft $P$. daedalea genome (REF). topGO ${ }^{52}$ was 478 used, with default settings, to perform GO term enrichment analyses on the genes of interest

479 list. Multiple testing correction was not applied on the resulting $p$-values as the tests were

480 considered to be non-independent ${ }^{52}$. GO terms with $p<0.05$ and occurring $\geq 5$ times in the background set were considered significant (Supplementary Data S7).

\section{Raw data}

484 Whole genome bisulphite sequencing data can be found in NCBI BioProject PRJNA430328.

Individual SRA accessions are listed in Supplementary Data S1c.

486 (Note: data is currently under embargo, and will appear as if it hasn't been uploaded on the

487 NCBI website. This embargo will be lifted upon publication)

\section{Code availability}

490 Python scripts and key input files are available at https://github.com/lyijin/pdae_dna_meth.

491 Explanatory notes for the scripts can be found in the repository.

\section{Acknowledgements}

494 We thank D. Abrego, G. Vaughan, and D. McParland for assistance with fieldwork, coral spawning, and the collection of environmental data, as well as the KAUST Sequencing Core

496 Facility for the sequencing of the libraries. This work was financially supported by KAUST

497 (sequencing and bioinformatics) and NYUAD (fieldwork and coral spawning). 
499 Author information

$500 \quad$ Affiliations

501 King Abdullah University of Science and Technology (KAUST), Red Sea Research Center

502 (RSRC), Biological and Environmental Sciences \& Engineering Division (BESE), Thuwal,

503 Saudi Arabia.

504 Yi Jin Liew, Xin Wang, Craig T. Michell, Manuel Aranda

505 Center for Genomics and Systems Biology, New York University Abu Dhabi, Abu Dhabi,

506 United Arab Emirates.

507 Emily J. Howells, John A. Burt, Youssef Idaghdour

\section{Contributions}

509 E.J.H, Y.I. and M.A. conceived and coordinated the project. M.A., X.W. and J.A.B provided

510 resources. E.J.H. collected samples from the wild, performed controlled crosses, and

511 extracted DNA from fixed samples. C.T.M. constructed libraries for WGBS and RNA-seq.

512 Y.J.L., Y.I. and M.A. analysed data. Y.J.L., E.J.H. and M.A. wrote the manuscript. All

513 authors read and approved the manuscript.

\section{Competing financial interests}

515 The authors declare no competing financial interests.

\section{Corresponding author}

517 Correspondence to: Manuel Aranda. 
519 Supplementary information

520 Supplementary Table S1. Age estimates of Platygyra daedalea colonies from Abu Dhabi.

521 Colony radius-age relationships established for $P$. sinensis from the Great Barrier Reef ${ }^{53}$

522 were applied to local data as this closely related species has identical growth morphology to

523 P. daedalea. Radial size gains were adjusted to reflect a $33 \%$ slower growth rate in Abu

524 Dhabi $P$. daedalea ${ }^{54}$ and applied to the sizes of colonies measured in the population $(n=$ $525140)$.

\begin{tabular}{ccccc} 
& $\begin{array}{c}\boldsymbol{P} \text {. sinensis } \\
\text { growth rates } \\
\text { (years per cm) }\end{array}$ & $\begin{array}{c}\boldsymbol{P} \text {. daedalea } \\
\text { growth rates } \\
\text { (years per cm, } \\
\text { adjusted) }\end{array}$ & $\begin{array}{c}\boldsymbol{P} \text {. daedalea } \\
\text { colony radius } \\
\text { (cm) }\end{array}$ & $\begin{array}{c}\boldsymbol{P} \text {. daedalea colony } \\
\text { age estimates } \\
\text { (mean, min-max) }\end{array}$ \\
\hline $\begin{array}{c}\text { Mean } \\
\text { Minimum }\end{array}$ & 1.9 & 2.5 & 12.8 & $32(24-41)$ \\
Maximum & 1.4 & 1.9 & 1.25 & $3(2-4)$ \\
& 2.4 & 3.2 & 47.8 & $120(91-153)$
\end{tabular}

526

527

528

Supplementary Table S2. Temperature and salinity profiles of $\boldsymbol{P}$. daedalea populations

529

in Abu Dhabi and Fujairah. Temperatures summaries were calculated from daily means

530 from in situ temperature loggers (HOBO pendant) attached to the reef substrate (Abu Dhabi:

531 2010-2014; Fujairah; 2012-2014). Salinity summaries were calculated from ad hoc

532 measurements (YSI multi-probe) taken in situ within $0.5 \mathrm{~m}$ of the substrate (Abu Dhabi:

$5332013-2017, n=23$; Fujairah; 2013-2015, $n=9$ ).

\begin{tabular}{ccc} 
Temperature $\left({ }^{\circ} \mathbf{C}\right)$ & Abu Dhabi & Fujairah \\
\hline Annual mean & 28.1 & 27.4 \\
Maximum & 35.7 & 33.3 \\
Minimum & 18.4 & 22.0 \\
Annual range & 17.3 & 11.3 \\
& & \\
Salinity (psu) & Abu Dhabi & Fujairah \\
\hline Mean & 42.1 & 37.7 \\
Maximum & 45.9 & 39.4 \\
Minimum & 39.6 & 35.5
\end{tabular}




\section{References}

536 1. Jablonka, E. \& Raz, G. Transgenerational epigenetic inheritance: prevalence, mechanisms, and implications for the study of heredity and evolution. Q Rev Biol 84, 131-176 (2009).

539

2. Lamke, J. \& Baurle, I. Epigenetic and chromatin-based mechanisms in environmental stress adaptation and stress memory in plants. Genome Biol 18, 124 (2017).

541

542

543

544

545

3. Lim, J. P. \& Brunet, A. Bridging the transgenerational gap with epigenetic memory. Trends Genet 29, 176-186 (2013).

4. Heard, E. \& Martienssen, R. A. Transgenerational epigenetic inheritance: myths and mechanisms. Cell 157, 95-109 (2014).

5. Moberg, F. \& Folke, C. Ecological goods and services of coral reef ecosystems. Ecol Econ 29, 215-233 (1999).

6. Hughes, T. P. et al. Spatial and temporal patterns of mass bleaching of corals in the Anthropocene. Science 359, 80-83 (2018).

7. Hughes, T. P. et al. Global warming and recurrent mass bleaching of corals. Nature 543, 373-377 (2017).

8. van Oppen, M. J., Oliver, J. K., Putnam, H. M. \& Gates, R. D. Building coral reef resilience through assisted evolution. Proc Natl Acad Sci U S A 112, 2307-2313 (2015).

9. Torda, G. et al. Rapid adaptive responses to climate change in corals. Nat Clim Change 7, 627-636 (2017).

10. Liew, Y. J. et al. Epigenome-associated phenotypic acclimatization to ocean acidification in a reef-building coral. bioRxiv (2017).

11. Dixon, G. B., Bay, L. K. \& Matz, M. V. Patterns of gene body methylation predict coral fitness in new environments. bioRxiv (2017).

12. Li, Y. et al. DNA methylation regulates transcriptional homeostasis of algal endosymbiosis in the coral model <em>Aiptasia</em>. bioRxiv (2018).

13. Veron, J. E. N. Corals of the World. Vol. 1-3 (Australian Institute of Marine Science, 2000).

14. Darling, E. S. et al. Evaluating life-history strategies of reef corals from species traits. Ecol Lett 15, 1378-1386 (2012).

15. Bauman, A. G., Baird, A. H. \& Cavalcante, G. H. Coral reproduction in the world's warmest reefs: southern Persian Gulf (Dubai, United Arab Emirates). Coral Reefs 30, 405-413 (2011).

16. Coles, S. L. \& Riegl, B. M. Thermal tolerances of reef corals in the Gulf: a review of the potential for increasing coral survival and adaptation to climate change through assisted translocation. Mar Pollut Bull 72, 323-332 (2013).

17. Howells, E. J. et al. Species-specific trends in the reproductive output of corals across environmental gradients and bleaching histories. Mar Pollut Bull 105, 532-539 (2016).

18. Dixon, G. B., Bay, L. K. \& Matz, M. V. Bimodal signatures of germline methylation are linked with gene expression plasticity in the coral Acropora millepora. BMC Genomics 15, 1109 (2014).

19. Dimond, J. L. \& Roberts, S. B. Germline DNA methylation in reef corals: patterns and potential roles in response to environmental change. Mol Ecol 25, 1895-1904 (2016). 
20. Wang, X. et al. Function and evolution of DNA methylation in Nasonia vitripennis. PLoS Genet 9, e1003872 (2013).

21. Lyko, F. et al. The honey bee epigenomes: differential methylation of brain DNA in queens and workers. PLoS Biol 8, e1000506 (2010).

22. Glastad, K. M., Gokhale, K., Liebig, J. \& Goodisman, M. A. The caste- and sexspecific DNA methylome of the termite Zootermopsis nevadensis. Sci Rep 6, 37110 (2016).

23. Putnam, H. M., Davidson, J. M. \& Gates, R. D. Ocean acidification influences host DNA methylation and phenotypic plasticity in environmentally susceptible corals. Evol Appl 9, 1165-1178 (2016).

24. Nakamura, N. Ubiquitination regulates the morphogenesis and function of sperm organelles. Cells 2, 732-750 (2013).

25. Song, W. H., Yi, Y. J., Sutovsky, M., Meyers, S. \& Sutovsky, P. Autophagy and ubiquitin-proteasome system contribute to sperm mitophagy after mammalian fertilization. Proc Natl Acad Sci U S A 113, E5261-5270 (2016).

26. Buscà, R., Pouysségur, J. \& Lenormand, P. ERK1 and ERK2 Map Kinases: Specific Roles or Functional Redundancy? Front Cell Dev Biol 4 (2016).

27. Grasso, L. C. et al. The biology of coral metamorphosis: molecular responses of larvae to inducers of settlement and metamorphosis. Dev Biol 353, 411-419 (2011).

28. Hayward, D. C. et al. Differential gene expression at coral settlement and metamorphosis--a subtractive hybridization study. PLoS One 6, e26411 (2011).

29. Imbs, A. B. \& Yakovleva, I. M. Dynamics of lipid and fatty acid composition of shallow-water corals under thermal stress: an experimental approach. Coral Reefs $\mathbf{3 1}$, 41-53 (2012).

30. Tolosa, I., Treignier, C., Grover, R. \& Ferrier-Pages, C. Impact of feeding and shortterm temperature stress on the content and isotopic signature of fatty acids, sterols, and alcohols in the scleractinian coral Turbinaria reniformis. Coral Reefs 30, 763-774 (2011).

31. Hillyer, K. E., Tumanov, S., Villas-Boas, S. \& Davy, S. K. Metabolite profiling of symbiont and host during thermal stress and bleaching in a model cnidariandinoflagellate symbiosis. J Exp Biol 219, 516-527 (2016).

32. Rosic, N. N. \& Dove, S. Mycosporine-like amino acids from coral dinoflagellates. Appl Environ Microbiol 77, 8478-8486 (2011).

33. Xu, C. \& Ng, D. T. Glycosylation-directed quality control of protein folding. Nat Rev Mol Cell Biol 16, 742-752 (2015).

34. Schweinsberg, M., Pech, R. A. G., Tollrian, R. \& Lampert, K. P. Transfer of intracolonial genetic variability through gametes in Acropora hyacinthus corals. Coral Reefs 33, 77-87 (2014).

35. Schweinsberg, M., Weiss, L. C., Striewski, S., Tollrian, R. \& Lampert, K. P. More than one genotype: how common is intracolonial genetic variability in scleractinian corals? Mol Ecol 24, 2673-2685 (2015).

36. Barfield, S., Aglyamova, G. V. \& Matz, M. V. Evolutionary origins of germline segregation in Metazoa: evidence for a germ stem cell lineage in the coral Orbicella faveolata (Cnidaria, Anthozoa). Proc Biol Sci 283 (2016).

37. Boyko, A. et al. Transgenerational adaptation of Arabidopsis to stress requires DNA methylation and the function of Dicer-like proteins. PLoS One 5, e9514 (2010).

38. Jiang, L. et al. Sperm, but not oocyte, DNA methylome is inherited by zebrafish early embryos. Cell 153, 773-784 (2013). 
39. Potok, M. E., Nix, D. A., Parnell, T. J. \& Cairns, B. R. Reprogramming the maternal zebrafish genome after fertilization to match the paternal methylation pattern. Cell 153, 759-772 (2013).

40. Wang, L. et al. Epigenetic regulation of left-right asymmetry by DNA methylation. EMBO J 36, 2987-2997 (2017).

41. Hofmeister, B. T., Lee, K., Rohr, N. A., Hall, D. W. \& Schmitz, R. J. Stable inheritance of DNA methylation allows creation of epigenotype maps and the study of epiallele inheritance patterns in the absence of genetic variation. Genome Biol 18, 155 (2017).

42. Lauss, K. et al. Parental DNA Methylation States Are Associated with Heterosis in Epigenetic Hybrids. Plant Physiol 176, 1627-1645 (2018).

43. van Oppen, M. J. H. et al. Shifting paradigms in restoration of the world's coral reefs. Glob Chang Biol 23, 3437-3448 (2017).

44. Howells, E. J., Abrego, D., Vaughan, G. O. \& Burt, J. A. Coral spawning in the Gulf of Oman and relationship to latitudinal variation in spawning season in the northwest Indian Ocean. Sci Rep 4, 7484 (2014).

45. Martin, M. Cutadapt removes adapter sequences from high-throughput sequencing reads. EMBnet.journal 17, pp. 10-12 (2011).

46. Krueger, F. \& Andrews, S. R. Bismark: a flexible aligner and methylation caller for Bisulfite-Seq applications. Bioinformatics 27, 1571-1572 (2011).

47. Bhatia, G., Patterson, N., Sankararaman, S. \& Price, A. L. Estimating and interpreting FST: the impact of rare variants. Genome Res 23, 1514-1521 (2013).

48. Shapiro, S. S. \& Wilk, M. B. An Analysis of Variance Test for Normality (Complete Samples). Biometrika 52, 591-\& (1965).

49. Levene, H. in Contributions to Probability and Statistics Vol. 1 (ed I. Olkin) 278292 (Stanford University Press, 1960).

50. Benjamini, Y. \& Hochberg, Y. Controlling the False Discovery Rate - a Practical and Powerful Approach to Multiple Testing. J R Stat Soc Series B Stat Methodol 57, 289300 (1995).

51. Benjamini, Y. \& Yekutieli, D. The control of the false discovery rate in multiple testing under dependency. Ann Stat 29, 1165-1188 (2001).

52. Alexa, A., Rahnenfuhrer, J. \& Lengauer, T. Improved scoring of functional groups from gene expression data by decorrelating GO graph structure. Bioinformatics 22, 1600-1607 (2006).

53. Babcock, R. C. Comparative demography of three species of scleractinian corals using age- and size - dependent classifications. Ecol Monogr 61, 225-244 (1991).

54. Howells, E. J. et al. Species-specific coral calcification responses to the extreme environment of the southern Persian Gulf. Front Mar Sci 5, 56 (2018). 